\title{
Integrated West Nile Virus Surveillance in Harris County, Texas, 2003 to 2018
}

\author{
Leann Liu, Mustapha Debboun, Kasimu Muhetaer, Eric V. Bakota, Martin Reyna, Dana \\ Beckham, Les Becker, Umair Shah
}

DCCP/DCME, Harris County Public Health, Houston, Texas, United States

Objective

This abstract aims to: 1) describe human WNV infections in Harris County excluding the City of Houston, Texas, 2003 to 2018 ; 2) explore geographical distributions of WNV positive mosquito pools in relation to human cases; 3 ) provide a brief overview of the county's rigorous multidisciplinary WNV surveillance and control in mosquitoes and humans.

Introduction

West Nile virus (WNV) is the leading cause of autochthonous arboviral disease in the United States [1]. The virus is primarily spread to people through the bite of infected Culex species of mosquitos. WNV was first identified in Harris County, Texas, in 2002 [2]. Since then, the mosquito-borne virus has become endemic in the region, with surges in 2012 and 2014. Although majority of individuals infected are asymptomatic, WNV induced neuroinvasive infections often result in hospitalizations and adverse outcomes [3-7], thus may pose a significant concern in public health and healthcare. The Harris County Public Health (HCPH) Surveillance and Epidemiology Unit (SEU) conducts surveillance of WNV in humans, in collaboration with Mosquito and Vector Control Division (MVCD) that monitors the virus in mosquito populations and birds. Mosquito abatement activities are implemented in areas where positive mosquitoes and human cases are identified. In this integrated model, clusters of WNV positive mosquito pools in relation with human cases provide comprehensive surveillance data to guide targeted efforts of mosquito control, disease prevention, and community education.

\section{Methods}

Surveillance data of human WNV cases and WNV tested mosquito pools 2003 to 2018 in Harris County excluding the City of Houston were used for the analysis. Human cases included were confirmed and probable cases. Frequencies, percentages, ageadjusted annual average rates were used to describe the data.

Geographical locations of WNV positive mosquito pools and human cases were mapped and analyzed using ESRI ArcGIS to determine the spatial relationship between the positive mosquito pools and human cases. Space-Time analysis was performed on 16 years of human disease data using Scan statistics in SatScan ${ }^{\mathrm{TM}}$ to test the effect of time and identify significant geographical clusters of WNV cases over time, which revealed a statistically significant cluster in 2012 to 2014 in northwest of Harris County. Subsequently, human cases and WNV positive mosquito pools of 2012 to 2014 were selected for hotspot analysis to verify the results from Satscan analysis and visualize the geographical cluster. Human cases were aggregated into census tracts and analyzed by optimized hotspot method; the positive mosquito pools were geocoded using their intersection locations and analyzed by ESRI Getis-Ord Gi hotspot method.

\section{Results}

From 2003 to 2018, a total of 295 confirmed and probable human WNV cases were identified, including 217 neuroinvasive and 78 fever cases. The median age of patients was 58 years old; $64.8 \%$ were male. The onset of majority of the cases $(80.7 \%)$ concentrated in July to September. Among case-patients, $72.9 \%$ were hospitalized, and 3.4\% died. The annual average age-adjusted incidence rate of WNV was 0.7 per 100,000 during 2003 to 2018. However, the rate was 1.8 and

2.7 per 100,000 in 2012 and 2014, respectively. Over $68 \%$ of the cases clustered in west and northwest of the county.

The annual WNV positive mosquito pools ranged from $0.2 \%$ to $10.2 \%$ amongst the years. The number of WNV positive mosquito pools in 2014 was more than 4 times higher than the average number during those years, the highest record in the County.

SDS Annual Conference Proceedings 2019. This is an Open Access article distributed under the terms of the Creative Commons AttributionNoncommercial 4.0 Unported License (http://creativecommons.org/licenses/by-nc/3.0/), permitting all non-commercial use, distribution, and reproduction in any medium, provided the original work is properly cited. 
Hot spots of human cases and the positive mosquito pools were both identified in northwest Harris County. There was a significant geographical overlap between human cases and the positive mosquito pools.

The space-time analysis for the 16 years detected a significant high-risk cluster in 2012 to 2014 in northwest Harris County. Findings from optimized hotspot analysis on human cases are consistent with the results from SatScan analysis. Statistically significant hot spots of positive mosquito pools identified by Getis-Ord Gi hotspot analysis displayed highly overlay with the statistically significant cluster of human cases detected by Satscan space-time analysis.

\section{Conclusions}

WNV infection is underestimated. Studies suggest that every one case identified represent five infections [8,9]. Therefore, the actual number of cases is likely substantially higher. Clinicians should be alerted in WNV season and consider testing and reporting as early as possible, especially in neuroinvasive patients. The public should be encouraged to utilize personal protection, particularly during peak seasons. This analysis shows that GIS mapping and optimized hotspot analysis of WNV human cases in relation to positive mosquito pools can provide statistical evidences of areas most affected, thus inform targeted mosquito control, prevention and education strategies in people living in areas with high virus activities.

\section{Acknowledgement}

We would like to acknowledge the colleagues in HCPH SEU and MVCD for their contributions to WNV surveillance.

\section{References}

1. Reimann CA, Hayes EB, DiGuiseppi C, et al. 2008. Epidemiology of neuroinvasive arbovrial disease in the United States, 1999-2007. Am J Trop Med Hyg. 79, 974-79. PubMed https://doi.org/10.4269/ajtmh.2008.79.974

2. Kristy M. Lillibridge, Ray Parsons, Yvonne Randle, et al. The 2002 introduction of West Nile virus into Harris county, Texas, an area historically endemic for St. Louis encephalitis

3. Mostashari F, Bunning ML, Kitsutani PT, et al. 2001. Epidemic West Nile encephalitis, New York, 1999: results of a household-based seroepidemiological survey. Lancet. 358, 261-64. PubMed https://doi.org/10.1016/S0140-6736(01)05480$\underline{0}$

4. Busch MP, Wright DJ, Custer B, et al. 2006. West Nile virus infections projected from blood donor screening data, United States, 2003. Emerg Infect Dis. 12, 395-402. PubMed https://doi.org/10.3201/eid1205.051287

5. Carson PJ, Borchardt SM, Custer B, et al. 2012. Neuroinvasive disease and West Nile virus infection, North Dakota, USA, 1999-2008. Emerg Infect Dis. 18, 684-86. PubMed https://doi.org/10.3201/eid1804.111313

6. Sejvar JJ, Haddad MB, Tierney BC, et al. 2003. Neurologic Manifestations and outcome of west nile virus infection. JAMA. 290(4), 511-15. PubMed https://doi.org/10.1001/jama.290.4.511

7. The outbreak of west nile virus infection in the New York City area in 1999

8. Surveillance for Human West Nile Virus Disease --- United States. 2010 / 59. 1999_2008. MMWR. SS02, 1-17. PubMed

9. West Nile Virus and Other Arboviral Diseases — United States. 2013 / 62. 2012. MMWR. (25), 513-17. PubMed 